\title{
Constrained optimal control of stochastic switched affine systems using randomization
}

\author{
Kostas Margellos, Alessandro Falsone, Simone Garatti and Maria Prandini
}

\begin{abstract}
We consider a finite-horizon optimal control problem for a switched affine system with controlled switches, affected by uncertainty and subject to input and/or state constraints. We show how the logical statements that govern the underlying switching mechanism can be transformed into robust mixed-integer inequalities, leading to an infinite dimensional linear program with robust constraints. Following a randomized methodology, based on enforcing the constraints only on a finite number of uncertainty instances/scenarios, we relax the infinite dimensional program to a mixed-integer linear program, which is amenable to existing numerical tools. We establish a probabilistic link between the infinite dimensional robust program and its scenario-based relaxation, showing that the optimal solution of the latter is feasible, in a probabilistic sense, for the former.
\end{abstract}

\section{INTRODUCTION}

Switched systems have been studied extensively in the dynamics and control literature due to their ability to capture the behavior of complex dynamical systems like those arising in ground transportation [1], [2], air traffic management [3], [4] and smart grid applications [5]. Such systems involve switching among different modes of operation, where at each mode the system evolves continuously. A formal analysis for various classes of switched dynamical systems (hybrid systems at their full level of generality), covering aspects ranging form stability and controller synthesis to verification, can be found in [6-8].

The interplay between continuous evolution and the underlying switching mechanism renders the analysis and control of general switched systems challenging. However, certain classes of switched systems admit tractable reformulations, allowing the use of existing numerical tools for analysis and controller synthesis. Discrete-time, switched systems with affine continuous dynamics at each mode of operation exhibit a prominent role among such problem classes. This is due to the fact that they can approximate the evolution of systems with nonlinear dynamics, and it has been shown that such systems are equivalent with mixed logical dynamical systems, for which efficient analysis and synthesis tools have been developed [9], [10].

Research was supported by the European Commission, H2020, under the project UnCoVerCPS, grant number 643921.

K. Margellos is with the Department of Engineering Science, University of Oxford, Parks Road, Oxford, OX1 3PJ, United Kingdom, e-mail: kostas.margellosdeng.ox.ac.uk

A. Falsone, S. Garatti and M. Prandini are with the Dipartimento di Elettronica Informazione e Bioingegneria, Politecnico di Milano, Piazza Leonardo da Vinci 32, 20133 Milano, Italy, email: $\quad$ alessandro.falsone, simone.garatti, maria.prandini\}@polimi.it
In the deterministic case, and due to the equivalence between switched affine systems and mixed logical dynamical systems, optimal control and verification problems for switched systems with affine dynamics can be recast as mixed integer optimization programs [9], [11], [12]. The discrete variables are introduced to encode the logical implications that are inherent in the switching mechanism. In the case, however, where the continuous dynamics are stochastic (i.e. they are affected by uncertainty), optimal control of stochastic switched systems becomes a more challenging task. From a verification point of view, typically under a reachability objective, significant contributions have been made [13], [14]. From an optimal control perspective, and in the case where uncertainty has a bounded support, an approach based on dynamic programming has been proposed in [15], [16]. If uncertainty is of unbounded support, but follows a Gaussian distribution, the authors of [17] follow a linear quadratic gaussian (LQG) based control procedure, whereas in [18] an algorithm based on semidefinite programming is proposed. However, these approaches do not capture the case where constraints on the state of the underlying dynamical system are imposed. Therefore, optimal control problems for switched affine systems affected by a possibly unbounded uncertainty, while taking state constraints into account, requires additional investigation.

In this paper we deal with the problem of finite-horizon optimal control of switched systems with affine dynamics, affected by uncertainty and subject to input and/or state constraints. Our contributions can be summarized as follows: 1) We focus on the case where switches are not autonomous but controlled, and extend the work of [9], showing how the logical statements that govern the underlying switching mechanism can be transformed into robust mixed-integer inequalities, capturing the case where possibly different input and/or state constraints per mode of operation may be present. We formulate the problem as an infinite dimensional linear program subject to robust constraints. 2) We relax the resulting program by means of a randomized methodology, that is based on enforcing the constraints only on a finite number of uncertainty instances, referred to as scenarios [19-22], rendering it a mixed-integer linear program that is amenable to existing numerical tools. Since the infinite dimensional program involved optimizing over functions, we employ the recent results of [23], appropriately extended to account for the presence of discrete variables, and establish a probabilistic link between this problem and its scenariobased relaxation. In particular, we show that the optimal control sequence calculated on the basis of a certain set of 
scenarios is feasible, in a probabilistic sense, for the infinite dimensional robust program.

The paper is structured as follows. In Section [II we introduce the class of stochastic switched affine systems under consideration and show how logical statements can be transformed into robust mixed-integer inequalities. In Section III we formulate an optimal control problem, relax it by means of a scenario-based methodology and establish a probabilistic link between these two problems. Finally Section IV concludes the paper and provides some directions for future work.

\section{SWITCHED SYSTEM}

\section{A. Dynamics and constraints}

We consider a discrete-time, switched affine system affected by uncertainty. To this end, for any time instance $k \in \mathbb{N}$, for all $i=1, \ldots, m$, let

$$
\begin{aligned}
z_{k}^{i}=1 & \Rightarrow \\
x_{k+1} & =A_{k}^{i}\left(w_{k}\right) x_{k}+B_{k}^{i}\left(w_{k}\right) u_{k}+v_{k}^{i}\left(w_{k}\right),
\end{aligned}
$$

with

$$
\sum_{i=1}^{m} z_{k}^{i}=1
$$

For every time instance $k, x_{k} \in \mathbb{R}^{n_{x}}$ is the state vector, $u_{k} \in$ $\mathbb{R}^{n_{u}}$ is the control input vector and $w_{k} \in W_{k} \subseteq \mathbb{R}^{n_{w}}$ is an uncertainty vector that encodes stochastic disturbances that may affect the system. Variables $z_{k}^{i} \in\{0,1\}, i=1, \ldots, m$, are binary valued control variables and (2) ensures that, at every time instance $k$, exactly one of them takes the value 1 . The mapping $v_{k}^{i}(\cdot): W_{k} \rightarrow \mathbb{R}^{n_{x}}$ is a known function of the uncertainty. Since some components of $v_{k}^{i}(\cdot)$ may include constants, (1) represents the evolution of a switched affine system, where switches are controlled by the choice of the binary variables, which can be thought of as discrete inputs. The system matrices are of appropriate dimension, i.e. for each $k$, for all $i=1, \ldots, m$, for all $w_{k} \in W_{k}, A_{k}^{i}\left(w_{k}\right) \in$ $\mathbb{R}^{n_{x} \times n_{x}}$ and $B_{k}^{i}\left(w_{k}\right) \in \mathbb{R}^{n_{x} \times n_{u}}$, where it is to be understood that the elements of $A_{k}^{i}\left(w_{k}\right)$ and $B_{k}^{i}\left(w_{k}\right)$ are functions of $w_{k}$.

By (1), according to the choice of $z_{k}^{i}$ a different set of dynamics is enabled. We say that at time $k$ the system operates at mode $i$ if the $i$-th set of dynamics is active, i.e. if $z_{k}^{i}=1$. At each mode of operation the state and input of the system may be subject to different physical and/or technological constraints. For each $k$, let $W_{\vec{k}}=\times_{j=0}^{k} W_{j}$, and denote by $w_{\vec{k}}=\left(w_{0}, \ldots, w_{k}\right) \in W_{\vec{k}}$ the collection of all uncertainty vectors up to time $k$. Similarly, we write $u_{\vec{k}} \in \mathbb{R}^{(k+1) n_{u}}$ and $z_{\vec{k}}^{i} \in\{0,1\}^{k+1}, i=1, \ldots, m$, for the continuous and discrete control inputs, respectively. For each $k$, and for all $i=1, \ldots, m$, the state and input constraints can be encoded by the following logical implication:

$$
\begin{aligned}
& z_{k}^{i}=1 \Rightarrow \\
& \quad f_{k}^{i}\left(x_{k}, u_{k}, w_{k}\right) \leq 0, \text { for all } w_{\vec{k}} \in W_{\vec{k}},
\end{aligned}
$$

where $f_{k}^{i}(\cdot, \cdot, \cdot): \quad \mathbb{R}^{n_{x}} \times \mathbb{R}^{n_{u}} \times W_{k} \rightarrow \mathbb{R}$. The subscript (superscript) $k(i)$ in $f_{k}^{i}$ is introduced to encode the fact that the system constraints may be different according to the time instance $k$ (the mode $i$ ). Note that the fact that a single constraint is considered is without loss of generality. In case of multiple constraints, $f_{k}^{i}(\cdot, \cdot, \cdot)$ can be taken to be the maximum among all involved constraint functions. The constraint in (3) is a robust constraint. Since $x_{k}$ depends on $u_{\overrightarrow{k-1}}, z_{\overrightarrow{k-1}}^{i}, i=1, \ldots, m$, and $w_{\overrightarrow{k-1}}$ due to the recursion in (1), $f_{k}^{i}\left(x_{k}, u_{k}, w_{k}\right) \leq 0$ should be satisfied not only for all $w_{k} \in W_{k}$, but for all $w_{\vec{k}} \in W_{\vec{k}}$, i.e. for all $w_{j} \in W_{j}$, $j=0, \ldots, k$.

We impose the following assumption:

Assumption 1: For each $k \in \mathbb{N}$, for all $i=1, \ldots, m$, $f_{k}^{i}(\cdot, \cdot, \cdot): \mathbb{R}^{n_{x}} \times \mathbb{R}^{n_{u}} \times W_{k} \rightarrow \mathbb{R}$ is jointly affine with respect to its first two arguments and continuous with respect to its last one. Moreover, $A_{k}^{i}(\cdot): W_{k} \rightarrow \mathbb{R}^{n_{x} \times n_{x}}$, $B_{k}^{i}(\cdot): \quad W_{k} \rightarrow \mathbb{R}^{n_{x} \times n_{u}}$ and $v_{k}^{i}(\cdot): W_{k} \rightarrow \mathbb{R}^{n_{x}}$ are continuous (elementwise) with respect to their argument.

Assumption 11 requires the constraint functions to be jointly affine with respect to state and continuous input vectors (hence also continuous), but allows for an arbitrary dependence (apart from continuity) with respect to the uncertainty. This is also the case for the dependency of the system dynamics with respect to the uncertainty. The affine structure is needed to facilitate the development of a tractable optimization program in Section III, whereas the continuity assumption is a technical requirement needed only to guarantee existence of the optimizers in problems (6), 10 and 111 of the next subsections. Note that we do not impose additional structure on the dependence of the constraint functions with respect to the uncertainty, since this is treated as parameter in the scenario-based approach adopted in Section III-B.

The switching mechanism encoded by (1)-(3) captures a wide class of problem structures, however, the implication in $[3$ is only one directional $(\Rightarrow)$; this allows us to capture controlled switches and encode different constraints per mode of operation, but excludes systems with switches that are triggered autonomously when the state of the system enters a certain partition of the space. This would require the opposite implication $(\Leftarrow)$ in $(3)$ to be also valid. The analysis of Section II-B, however, is not applicable in such cases, since it is not straightforward how to reformulate such an implication in a form that can be amenable to computational tools.

\section{B. Reformulating logical implications to mixed-integer equalities/inequalities}

Consider first the switched system dynamics in (1). It can be easily observed that, for each $k$, and due to (2), (1) can be equivalently written as

$$
x_{k+1}=\sum_{i=1}^{m}\left(A_{k}^{i}\left(w_{k}\right) x_{k}+B_{k}^{i}\left(w_{k}\right) u_{k}+v_{k}^{i}\left(w_{k}\right)\right) z_{k}^{i} .
$$

We will now reformulate the logical implication in (3) to a robust, mixed-integer constraint. To this end, we follow the 
procedure outlined in [9], to equivalently represent logical statements by mixed-integer inequalities. We impose the following assumption.

Assumption 2: For each $k \in \mathbb{N}$, for all $i=1, \ldots, m$, the set

$$
\begin{aligned}
C_{k}^{i}=\left\{(x, u, w) \in \mathbb{R}^{n_{x}} \times \mathbb{R}^{n_{u}}\right. & \times W_{k}: \\
& \left.f_{k}^{i}(x, u, w) \leq 0\right\},
\end{aligned}
$$

is non-empty and compact.

For each $k$, for all $i=1, \ldots, m$, let $M_{k}^{i} \in \mathbb{R}$ denote the maximum value $f_{k}^{i}(x, u, w)$ can achieve over $(x, u, w) \in$ $\bigcup_{i=1}^{m} C_{k}^{i}$, i.e. over all possible values that $(x, u, w)$ may take irrespective of the mode that is active at time $k$. This is given by means of the following optimization problem

$$
M_{k}^{i}=\max _{(x, u, w) \in \bigcup_{i=1}^{m} C_{k}^{i}} f_{k}^{i}(x, u, w) .
$$

Under Assumptions 1 and 2, $M_{k}^{i}$ exists and is finite (maximum of a continuous function over a compact domain). However, problem (6) may be difficult to solve in practice since it requires maximizing a possibly non-concave (with respect to $w$ ) function over a compact, under Assumption 2. domain of arbitrary geometry. Its solution can be thus computed only in particular cases, e.g., when $\bigcup_{i=1}^{m} C_{k}^{i}$ is a polytope or ellipsoid, and $f_{k}^{i}(\cdot, \cdot, \cdot)$ is concave with respect to its last argument (it is affine with respect to $x, u$ under Assumption (1). A viable alternative that is often used in practice is to set $M_{k}^{i} \in \mathbb{R}$ to a very large constant, chosen

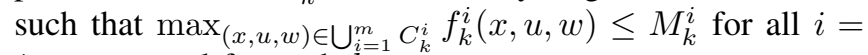
$1, \ldots, m$, and for each $k$.

By [9] (Section 2), we then have that for each $k$, for all $i=1, \ldots, m$, (3) is equivalent to

$$
\begin{aligned}
f_{k}^{i}\left(x_{k}, u_{k}, w_{k}\right) \leq M_{k}^{i}\left(1-z_{k}^{i}\right), & \\
& \text { for all } w_{\vec{k}} \in W_{\vec{k}},
\end{aligned}
$$

The equivalence between (3) and (7) can be seen by inspecting that $z_{k}^{i}=1$ in (7) implies that $f_{k}^{i}\left(x_{k}, u_{k}, w_{k}\right) \leq 0$, whereas if $z_{k}^{i}=0$ we have that $f_{k}^{i}\left(x_{k}, u_{k}, w_{k}\right) \leq M_{k}^{i}$ for all $w_{\vec{k}} \in W_{\vec{k}}$, which, by the definition of $M_{k}^{i}$ is satisfied for all admissible values of $x_{k}, u_{k}$, so no additional constraints are imposed. The reformulation in (7) serves then as a big- $M$ type of procedure.

Under Assumptions 1 and 2, the logical implications in (1), (3), are equivalent to the mixed integer equality (4) and inequality (7), respectively. However, (4) involves bilinear terms that impose the following challenge: If, by propagating (4) in time, $x(k)$ is written as a function of the initial state $x_{0}$, $u_{\overrightarrow{k-1}}, z_{\overrightarrow{k-1}}^{i}, i=1, \ldots, m$, and $w_{\overrightarrow{k-1}}$, and is substituted in 7), the resulting constraint would no longer be affine with respect to $u_{\overrightarrow{k-1}}$. The latter would raise tractability issues when formulating the optimization program of Section III. In view of this, we show how this problem can be alleviated in the next subsection.

\section{Dealing with bilinear terms}

Due to the fact that the bilinear terms in (4) do not involve products between the state and the continuous input variables, (4) can be equivalently written in a form that does not include such terms. To this end, for each $k$, for all $i=1, \ldots, m$, consider a function $y_{k}^{i}(\cdot): W_{\vec{k}} \rightarrow \mathbb{R}^{n_{x}}$ such that for all $w_{\vec{k}} \in W_{\vec{k}}$,

$$
y_{k}^{i}\left(w_{\vec{k}}\right)=\left(A_{k}^{i}\left(w_{k}\right) x_{k}+B_{k}^{i}\left(w_{k}\right) u_{k}+v_{k}^{i}\left(w_{k}\right)\right) z_{k}^{i},
$$

where the dependency of $y_{k}^{i}(\cdot)$ on the entire uncertainty history $w_{\vec{k}}$ and not only on $w_{k}$ is due to $x_{k}$.

Following the analysis of [9] (Section 3), (8) can be equivalently represented by the following set of robust constraints. For each $k$, for all $i=1, \ldots, m$, and for all $w_{\vec{k}} \in W_{\vec{k}}$,

$$
\begin{aligned}
& y_{k}^{i}\left(w_{\vec{k}}\right) \leq \bar{M}_{k}^{i} z_{k}^{i}, \\
& y_{k}^{i}\left(w_{\vec{k}}\right) \geq \underline{M}_{k}^{i} z_{k}^{i}, \\
& y_{k}^{i}\left(w_{\vec{k}}\right) \leq A_{k}^{i}\left(w_{k}\right) x_{k}+B_{k}^{i}\left(w_{k}\right) u_{k}+v_{k}^{i}\left(w_{k}\right)-\underline{M}_{k}^{i}\left(1-z_{k}^{i}\right), \\
& y_{k}^{i}\left(w_{\vec{k}}\right) \geq A_{k}^{i}\left(w_{k}\right) x_{k}+B_{k}^{i}\left(w_{k}\right) u_{k}+v_{k}^{i}\left(w_{k}\right) \\
&-\bar{M}_{k}^{i}\left(1-z_{k}^{i}\right) .
\end{aligned}
$$

For each $k$, for all $i=1, \ldots, m, \bar{M}_{k}^{i}=$ $\left(\bar{M}_{k, 1}^{i}, \ldots, \bar{M}_{k, n_{x}}^{i}\right) \in \mathbb{R}^{n_{x}}, \underline{M}_{k}^{i}=\left(\underline{M}_{k, 1}^{i}, \ldots, \underline{M}_{k, n_{x}}^{i}\right) \in$ $\mathbb{R}^{n_{x}}$. For $j=1, \ldots, n_{x}, \bar{M}_{k, j}^{i}, \underline{M}_{k, j}^{i} \in \mathbb{R}$, denote the worst-case maximum and minimum value that $A_{k, j}^{i}(w) x_{k}+B_{k, j}^{i}(w) u_{k}+v_{k, j}^{i}(w)$ can achieve over $(x, u, w) \in \bigcup_{i=1}^{m} C_{k}^{i}$, where $A_{k, j}^{i}(w), B_{k, j}^{i}(w), v_{k, j}^{i}(w)$, denote the $j$-th row of $A_{k}^{i}(w), B_{k}^{i}(w)$ and $v_{k}^{i}(w)$, respectively. For $j=1, \ldots, n_{x}$, they are given by means of the following optimization problems

$$
\begin{aligned}
& \bar{M}_{k, j}^{i}=\max _{(x, u, w) \in \bigcup_{i=1}^{m} C_{k}^{i}} A_{k, j}^{i}(w) x+B_{k, j}^{i}(w) u+v_{k, j}^{i}(w), \\
& \underline{M}_{k, j}^{i}=\min _{(x, u, w) \in \bigcup_{i=1}^{m} C_{k}^{i}} A_{k, j}^{i}(w) x+B_{k, j}^{i}(w) u+v_{k, j}^{i}(w) .
\end{aligned}
$$

Under Assumption 2, for all $j=1, \ldots, n_{x}, \bar{M}_{k, j}^{i}, \underline{M}_{k, j}^{i}$ exist and are finite. However, as with $M_{k}^{i}$, determining them by means of the aforementioned optimization programs may be computationally challenging, apart form particular cases (e.g., when $\bigcup_{i=1}^{m} C_{k}^{i}$ is a polytope or ellipsoid). In practice, for each $k$, for all $i=1, \ldots, m$, for all $j=1, \ldots, n_{x}$, $\bar{M}_{k, j}^{i}$ is typically set to a large constant, chosen such that

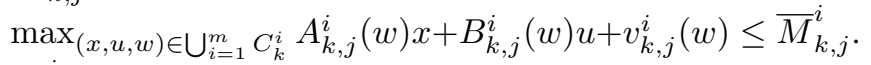
$\underline{M}_{k, j}^{i}$ is chosen analogously.

The equivalence between (8) and (9) can be seen by inspecting that if $z_{k}^{i}=1$, the last two constraints in (9) imply that $y_{k}^{i}\left(w_{\vec{k}}\right)=A_{k}^{i}\left(w_{k}\right) x_{k}+B_{k}^{i}\left(w_{k}\right) u_{k}+v_{k}^{i}\left(w_{k}\right)$, and the first two that $\underline{M}_{k}^{i} \leq y_{k}^{i}\left(w_{\vec{k}}\right)=A_{k}^{i}\left(w_{k}\right) x_{k}+B_{k}^{i}\left(w_{k}\right) u_{k}+$ $v_{k}^{i}\left(w_{k}\right) \leq \bar{M}_{k}^{i}$. The latter is trivially satisfied by the definition of $\bar{M}_{k}^{i}, \underline{M}_{k}^{i}$, since for each $k,\left(x_{k}, u_{k}, w_{k}\right) \in \bigcup_{i=1}^{m} C_{k}^{i}$. 
Under a similar reasoning, if $z_{k}^{i}=0$, the first two constraints in (9) imply that $y_{k}^{i}\left(w_{\vec{k}}\right)=0$ (hence the contribution of the corresponding term in (4) is zero), and, as a result, the last two lead to $\underline{M}_{k}^{i} \leq A_{k}^{i}\left(w_{k}\right) x_{k}+B_{k}^{i}\left(w_{k}\right) u_{k}+v_{k}^{i}\left(w_{k}\right) \leq \bar{M}_{k}^{i}$, which is trivially satisfied over $\bigcup_{i=1}^{m} C_{k}^{i}$ by the definition of $\bar{M}_{k}^{i}, \underline{M}_{k}^{i}$.

Under the assignment in (8), and due to (4),

$$
x_{k+1}=\sum_{i=1}^{m} y_{k}^{i}\left(w_{\vec{k}}\right) .
$$

By means of (12), substitute $x_{k}$ in (7) and 9). Notice that, under Assumption 11. for each $k$, for all $i=1, \ldots, m$, the constraints in (7) and (9) would have an affine dependence with respect to $u_{k}, z_{k}^{i}, y_{k}^{i}\left(w_{\vec{k}}\right)$ and $\left\{y_{k-1}^{i}\left(w_{\overrightarrow{k-1}}\right)\right\}_{i=1}^{m}$.

\section{OPTIMAL CONTROL PROBLEM}

\section{A. Problem set-up}

Let $T \in \mathbb{N}_{+}$be a finite time horizon. By appropriately choosing the continuous control inputs $u_{\overrightarrow{T-1}}$ and by switching among the $m$ modes of operation over the horizon $T$ via the discrete inputs $z_{\overrightarrow{T-1}}^{i}, i=1, \ldots, m$, we seek to optimize a certain performance criterion subject to the switching mechanism (1)-(3), with $k=0, \ldots, T-1$.

It was shown in Sections II-B and II-C that for all $k=0, \ldots, T-1$, for all $i=1, \ldots, m$, (1) and (3) are equivalent to (12) and (7), respectively. Due to this equivalence, the switching mechanism can be encoded by (2), (7) and (9), which due to (12) depend on $u_{k}, w_{k}, z_{k}^{i}$, $y_{k}^{i}\left(w_{\vec{k}}\right)$ and $\left\{y_{k-1}^{i}\left(w_{\overrightarrow{k-1}}\right)\right\}_{i=1}^{m}$ (see also discussion at the end of Section II-C). Collecting then all these constraints for all $k=0, \ldots, T-1$, and for all $i=1, \ldots, m$, and after some algebraic rearrangements, we can compactly represent them by

$$
g(u, z, y(w), w) \leq 0, \text { for all } w \in \mathbb{W}
$$

where $\mathbb{W}=W_{\overrightarrow{T-1}}, u=u_{\overrightarrow{T-1}} \in \mathbb{R}^{T n_{u}}, w=$ $w_{\overrightarrow{T-1}} \in \mathbb{W}$ and $z=\left(z_{\overrightarrow{T-1}}^{1}, \ldots, z_{\overrightarrow{T-1}}^{m}\right) \in\{0,1\}^{m T}$. The function $y(\cdot): \mathbb{W} \rightarrow \mathbb{R}^{m T n_{x}}$ is such that, for all $w \in \mathbb{W}, y(w)=\left(y^{1}(w), \ldots, y^{m}(w)\right)$, where $y^{i}(w)=$ $\left(y_{0}^{i}\left(w_{\overrightarrow{0}}\right), \ldots, y_{T-1}^{i}\left(w_{\overrightarrow{T-1}}^{\longrightarrow}\right)\right) \in \mathbb{R}^{T n_{x}}, i=1, \ldots, m$. Note that under Assumption 1, and due to the fact that bilinearities were resolved in Section II-C $g(u, z, y(w), w)$ is affine with respect to its first three arguments, whereas its dependence on $w$ is arbitrary.

Let $c=\left(c_{0}, \ldots, c_{T-1}\right) \in \mathbb{R}^{T n_{u}}$, where $c_{k} \in \mathbb{R}^{n_{u}}$, $k=0, \ldots, T-1$, and consider the following finite-horizon optimization program.

$$
\begin{aligned}
& \mathcal{P}_{\mathbb{W}}: \min _{\substack{u \in \mathbb{R}^{T n_{u}}, z \in\{0,1\}^{m T} \\
y(\cdot): \mathbb{W} \rightarrow \mathbb{R}^{m T n_{x}}}} c^{\top} u \\
& \text { subject to } \\
& g(u, z, y(w), w) \leq 0, \text { for all } w \in \mathbb{W} .
\end{aligned}
$$

The subscript $\mathbb{W}$ in $\mathcal{P}_{\mathbb{W}}$ is introduced to emphasize the presence of robust constraints. The fact that a linear objective function involving only the continuous control inputs is considered is without loss of generality. A more general objective function (e.g., worst-case performance metric with respect to the uncertainty) could be recast in this framework by means of an epigraphic reformulation. Note that by appropriately selecting $f_{T-1}^{i}(\cdot, \cdot, \cdot)$ (incorporated in the definition of $g(\cdot, \cdot, \cdot, \cdot)$ ) so that it depends on $x_{T}=A_{T-1}^{i} x_{T-1}+$ $B_{T-1}^{i} u_{T-1}+v_{T-1}^{i}\left(w_{T-1}\right)$, a terminal state constraint can be imposed.

Problem $\mathcal{P}_{\mathbb{W}}$ is a robust, mixed-integer linear program. There are, however, two main difficulties that prevent $\mathcal{P}_{\mathbb{W}}$ from being amenable to existing numerical tools. The first refers to the fact that it is an infinite dimensional optimization program since it involves optimizing over functions $y(\cdot)$. The second is the fact that the constraints in (15) should be robustly satisfied for the different values uncertainty may take in $\mathbb{W}$. However, the set $\mathbb{W}$ may not be known explicitly (e.g., only historical data for $w$ may be available), or, if known, it may be a continuous set, thus imposing tractability challenges. In the next subsections we show how to overcome these difficulties by exploiting scenario-based optimization [19], [20], [22].

\section{B. Scenario-based relaxation}

To deal with the robust constraint in $\widetilde{\mathcal{P}}_{\mathbb{W}}$, we propose a scenario-based relaxation [19], [20]. Consider first the following optimization program

$$
\begin{aligned}
& \widetilde{\mathcal{P}}_{\mathbb{W}}: \min _{u \in \mathbb{R}^{T n_{u}, z \in\{0,1\}^{m T}}} c^{\top} u \\
& \text { subject to } \\
& \min _{\widetilde{y} \in \mathbb{R}^{m T n_{x}}} g(u, z, \widetilde{y}, w) \leq 0 \text {, for all } w \in \mathbb{W} .
\end{aligned}
$$

It can be easily seen that problems $\mathcal{P}_{\mathbb{W}}$ and $\widetilde{\mathcal{P}}_{\mathbb{W}}$ are equivalent in the sense that they admit the same solutions for $u, z$ and the same optimal objective value. Compared to $\mathcal{P}_{\mathbb{W}}, \widetilde{\mathcal{P}}_{\mathbb{W}}$ is not an easier problem since it is not amenable to numerical tools due to the robust minimization problem that effectively appears in the constraints.

We consider a scenario-based relaxation of $\widetilde{\mathcal{P}}_{\mathbb{W}}$, where instead of enforcing the constraints for all values of $w$ in $W$, we enforce them only on $N$ realizations/scenarios of the uncertainty. Let $\left(w^{(1)}, \ldots, w^{(N)}\right) \in \mathbb{W}^{N}$ be a vector of $N$ identically and independently distributed (i.i.d.) scenarios $w^{(j)}, j=1, \ldots, N$, of the uncertainty. For each $j=$ $1, \ldots, N$, we introduce a different decision vector $\widetilde{y}^{(j)} \in$ $\mathbb{R}^{m T n_{x}}$, and consider the following optimization program.

$$
\begin{aligned}
& \widetilde{\mathcal{P}}_{N}: \min _{u \in \mathbb{R}^{T n_{u}}, z \in\{0,1\}^{m T}} c^{\top} u \\
& \text { subject to } \\
& \min _{\widetilde{y}^{(j)} \in \mathbb{R}^{m T n_{x}}} g\left(u, z, \widetilde{y}^{(j)}, w^{(j)}\right) \leq 0, \\
& \text { for all } j=1, \ldots, N \text {. }
\end{aligned}
$$

Problem $\widetilde{\mathcal{P}}_{N}$ is equivalent to the following minimization program, where the minimization in the constraint (19) is 
lifted in the objective (see 20, below).

$$
\begin{array}{cc}
\mathcal{P}_{N}: & \min \\
& \left\{\widetilde{y}^{(j)} \in \mathbb{R}^{m T n_{x}}\right\}_{j=1}^{N} \\
& c^{\top} u
\end{array}
$$

subject to

$$
g\left(u, z, \widetilde{y}^{(j)}, w^{(j)}\right) \leq 0, \text { for all } j=1, \ldots, N
$$

The subscript $N$ in $\widetilde{\mathcal{P}}_{N}, \mathcal{P}_{N}$ is introduced to emphasize the dependency on the uncertainty scenarios. Due to the fact that $g\left(u, z, \widetilde{y}^{(j)}, w^{(j)}\right), j=1, \ldots, N$, is affine with respect to the decision variables, $\mathcal{P}_{N}$ is a mixed-integer linear program. As a byproduct of the scenario-based methodology, in contrast to $\mathcal{P}_{\mathbb{W}}, \mathcal{P}_{N}$ not only has a finite number of constraints, but also a finite number of decision variables. In particular, it is no longer required to optimize over functions $y(\cdot)$, but we need to introduce the additional decision vector $\widetilde{y}^{(j)}$ per scenario of the uncertainty. The computational limitation of this scenario-based approach is that not only the number of constraints, but also the number of decision variables grows linearly with the number of scenarios.

\section{Connection between $\mathcal{P}_{N}$ and $\mathcal{P}_{\mathbb{W}}$}

Problem $\mathcal{P}_{N}$ falls in the framework of [23] and could be thought of as a scenario program with certificates. In fact, we refer to variables $\widetilde{y}^{(j)} \in \mathbb{R}^{m T n_{x}}, j=1, \ldots, N$, as certificates, since, unlike $u$ and $z$, we are not interested in their specific value, but it suffices that one set of such variables that satisfies (21) exists. This can be also seem from the equivalence between $\mathcal{P}_{N}$ and $\mathcal{P}_{N}$, and explains why $\widetilde{y}^{(j)}$, $j=1, \ldots, N$, are treated differently from the other decision variables in the derivations below. We impose the following assumption.

Assumption 3: For any set $\left\{w^{(1)}, \ldots, w^{(N)}\right\} \subset \mathbb{W}$ of $N$ i.i.d. scenarios of $w \in \mathbb{W}, \mathcal{P}_{N}$ has a non-empty feasibility region and its minimizer exists and is unique.

A direct consequence of Assumption 3 is that for $\mathcal{P}_{N}$ to have a non-empty feasibility region, $\mathbb{W}$ should not be unbounded. The uniqueness part of Assumption 3 can be relaxed as shown in [20], e.g., by using a deterministic tiebreak rule in case of multiple solutions. Let $\mathbb{W}$ be endowed with a $\sigma$-algebra and assume that $w \in \mathbb{W}$ is distributed according to some fixed, but possibly unknown probability distribution $\mathbb{P}$, defined over this $\sigma$-algebra. Denote by $\mathbb{P}^{N}$ the corresponding product measure.

Denote by $u_{N}^{*}$ and $z_{N}^{*}$ the minimizers of $\mathcal{P}_{N}$, as far as the $u$ and $z$ variables are concerned. The subscript $N$ is introduced to emphasize the fact that these minimizers depend on the $N$ scenarios used to formulate $\mathcal{P}_{N}$, and will be different if another set of $N$ scenarios is considered instead. We then have the following proposition, which is in line with the proof of Theorem 1 in [23], and relies on Theorem 1 of [20], modified to account for the presence of binary variables using the results in [24], [25].

Proposition 1: Consider Assumptions 1 3 Fix $\epsilon \in(0,1)$, consider $\left\{w^{(j)}\right\}_{j=1}^{N}$ and formulate $\mathcal{P}_{N}$. With confidence at least $1-2^{m T} \sum_{k=0}^{m T n_{u}-1}\left(\begin{array}{c}N \\ k\end{array}\right) \epsilon^{k}(1-\epsilon)^{N-k}$, the minimizers
$u_{N}^{*}$ and $z_{N}^{*}$ of $\mathcal{P}_{N}$ are feasible for $\widetilde{\mathcal{P}}_{\mathbb{W}}$ with probability at least $1-\epsilon$, i.e.

$$
\begin{aligned}
& \mathbb{P}^{N}\left\{\left(w^{(1)}, \ldots, w^{(N)}\right) \in \mathbb{W}^{N}:\right. \\
& \left.\mathbb{P}\left\{w \in \mathbb{W}: \min _{\widetilde{y} \in \mathbb{R}^{m T n_{x}}} g\left(u_{N}^{*}, z_{N}^{*}, \widetilde{y}, w\right) \leq 0\right\} \geq 1-\epsilon\right\} \\
& \geq 1-2^{m T} \sum_{k=0}^{m T n_{u}-1}\left(\begin{array}{c}
N \\
k
\end{array}\right) \epsilon^{k}(1-\epsilon)^{N-k}
\end{aligned}
$$$$
\text { Proof: } \quad k=0 \text { The constraint function }
$$

$\min _{\widetilde{y} \in \mathbb{R}^{m T n_{x}}} g(u, z, \widetilde{y}, w)$ in (17) is convex (in fact it is affine due to Assumption [1] with respect to the $u$ variables. This follows from the fact that $g(u, z, \widetilde{y}, w)$ is jointly convex with respect to $u$ and $\widetilde{y}$, and minimizing only over $\widetilde{y}$ in (17) preserves convexity with respect to $u$ [26] (Chapter 3.2.5, p. 87).

Therefore, $\widetilde{\mathcal{P}}_{\mathbb{W}}$ is a robust, mixed-integer program, with a constraint function that is convex with respect to the continuous decision variables, and $\widetilde{\mathcal{P}}_{N}$ is a scenario program corresponding to $\widetilde{\mathcal{P}}_{\mathbb{W}}$. Under Assumption 3 , the result follows then directly by Theorem 3 of [24] (or equivalently Theorem 4.1 in [25]), according to which we have that, with confidence at least $1-2^{m T} \sum_{k=0}^{m T n_{u}-1}\left(\begin{array}{l}N \\ k\end{array}\right) \epsilon^{k}(1-\epsilon)^{N-k}$, the minimizers $u_{N}^{*}$ and $z_{N}^{*}$ of $\mathcal{P}_{N}$ (and hence also of $\widetilde{\mathcal{P}}_{N}$ ) are feasible for $\widetilde{\mathcal{P}}_{\mathbb{W}}$ with probability at least $1-\epsilon$. The statement follows then from the equivalence between $\widetilde{\mathcal{P}}_{N}$ and $\mathcal{P}_{N}$.

Proposition 1 shows that with certain confidence, the solution generated by the scenario-based optimization program $\mathcal{P}_{N}$ is feasible for $\widetilde{\mathcal{P}}_{\mathbb{W}}$, apart from a set with measure at most $\epsilon$. The latter, due to the equivalence between $\widetilde{\mathcal{P}}_{\mathbb{W}}$ and $\mathcal{P}_{\mathbb{W}}$, establishes a probabilistic link between $\mathcal{P}_{N}$ and $\mathcal{P}_{\mathbb{W}}$.

Note that Proposition 1 can be also interpreted as follows. Fix $\beta \in(0,1)$ and choose $N$ such that

$$
2^{m T} \sum_{k=0}^{m T n_{u}-1}\left(\begin{array}{l}
N \\
k
\end{array}\right) \epsilon^{k}(1-\epsilon)^{N-k} \leq \beta .
$$

We then have that with confidence at least $1-\beta, u_{N}^{*}$ and $z_{N}^{*}$ are feasible for $\widetilde{\mathcal{P}}_{\mathbb{W}}$ with probability at least $1-\epsilon$. We are interested in determining the minimum value of $N \in \mathbb{N}$ that satisfies (23) since this would lead to an optimization program with fewer constraints and decision variables. This can be computed by means of 23) via numerical inversion (e.g., using bisection). An explicit, albeit conservative, expression to compute $N$ is given by

$$
N \geq \frac{e}{e-1} \frac{1}{\epsilon}\left(m T n_{u}-1+m T \ln \frac{1}{\beta}\right) .
$$

The reader is referred to [24] (Theorem 3, Corollary 3), for more details on its derivation. In [24], however, 24] appears with 2 in place of $e /(e-1)$; the refinement of using $e /(e-1)$ instead is due to [27], [28]. Note that the presence of the term $2^{m T}$ in front of the summation in (23) dictates the number of possible binary combinations that may occur. This term would not be present if we only had continuous decision variables. In (24), we have the term $m T$ in front of the logarithm since $\ln \left(2^{m T} / \beta\right)=m T \ln (1 / \beta)$; see also [25] for a detailed discussion on this issue. Note that 24] is 
linear with respect to the decision variables (both continuous and discrete) and grows logarithmically with respect to $\beta$. The latter implies that $\beta$ can be set to very low values (i.e. we can claim the feasibility result of Proposition 11 with high confidence), without an unaffordable increase in $N$, and hence in the computational complexity of the resulting optimization program.

The number $m T n_{u}$ of continuous decision variables that appears in 23, 24, upper-bounds the number of support constraints for $\mathcal{P}_{N}$, a notion which is at the basis of the scenario approach theory [19], [20] (see [19] (Definition 4) for a formal definition of the support constraints). Using the results of [29], in certain cases tighter upper-bounds can be obtained, leading to tighter sample size bounds for $N$.

\section{CONCLUDING REMARKS}

In this paper we formulated a finite-horizon optimal control problem for a switched affine system with controlled switches, affected by uncertainty with a possibly unbounded support, and subject to input and/or state constraints. It was shown how to reformulate the logical statements at the basis of the switching mechanism to robust mixed-integer inequalities, resulting into an infinite dimensional linear program with robust constraints. The latter was relaxed by means of a randomized methodology, that was based on enforcing the constraints only on a finite number of uncertainty scenarios. It was then shown that the optimal solution of the resulting scenario program is feasible, in a probabilistic sense, for the infinite dimensional robust program.

Current work concentrates on extending the developed framework to capture the case where switches may be triggered autonomously when entering a certain partition of the state space. Moreover, we investigate the possibility of employing difference performance criteria, based on the expected value or some other risk metric of an objective function. From an application point of view, we investigate the potential of applying the developed methodology to extend the deterministic microgrid energy management problem of [5], [30] to the case where stochastic uncertainty is present.

\section{REFERENCES}

[1] J. Lygeros, D. Godbole, and S. Sastry, "Verified hybrid controllers for automated vehicles," IEEE Transactions on Automatic Control, vol. 43, no. 4 , pp. $522-539,1998$.

[2] M. Althoff, O. Stursberg, and M. Buss, "Safety assessment of autonomous cars using verification techniques," American Control Conference, pp. 4154 - 4159, 2007.

[3] K. Margellos and J. Lygeros, "Toward 4-D Trajectory Management in Air Traffic Control: A Study Based on Monte Carlo Simulation and Reachability Analysis," IEEE Transactions on Control Systems Technology, vol. 21, no. 5, pp. 1820 - 1833, 2013.

[4] M. Prandini and J. Hu, "Application of Reachability Analysis for Stochastic Hybrid Systems to Aircraft Conflict Prediction," IEEE Transactions on Automatic Control, vol. 54, no. 4, pp. 913 - 917, 2009.

[5] A. Parisio, E. Rikos, and L. Glielmo, "A model predictive control approach to microgrid operation management," IEEE Transactions on Control Systems Technology, vol. 22, no. 5, pp. 1813 - 1827, 2014.

[6] J. Hespanha, "Logic-based switching algorithms in control," Ph.D. Thesis, Yale University, 1998.

[7] D. Liberzon, "Switching in systems and control," Birkhauser, Boston, MA, 2003.
[8] J. Lygeros, S. Sastry, and C. Tomlin, "Hybrid Systems: Foundations, advanced topics and applications," Lecture Notes, 2012.

[9] A. Bemporad and M. Morari, "Control of systems integrating logic, dynamics, and constraints," Automatica, vol. 35, no. 3, pp. 407-427, 1999.

[10] W. P. M. H. Heemels, B. DeSchutter, and A. Bemporad, "Equivalence of hybrid dynamical models," Automatica, vol. 37, no. 7, pp. 1085 1091, 2001.

[11] A. Bemporad, G. Ferrari-Trecate, and M. Morari, "Observability and controllability of piecewise affine and hybrid systems," IEEE Transactions on Automatic Control, vol. 45, no. 10, pp. 1864 - 1876, 2000.

[12] A. Bemporad, F. Torrisi, and M. Morari, “ Optimization-based verification and stability characterization of piecewise affine and hybrid systems," Hybrid Systems Computation and Control Conference, eds. B. Krogh, N. Lynch, pp. $45-58,2000$.

[13] A. Abate, M. Prandini, J. Lygeros, and S. Sastry, "Probabilistic reachability and safety for controlled discrete time stochastic hybrid systems," Automatica, vol. 44, no. 11, pp. 2724 - 2734, 2008.

[14] S. Summers and J. Lygeros, "Verification of discrete time stochastic hybrid systems: A stochastic reach-avoid decision problem," Automatica, vol. 46, no. 12, pp. $1951-1961,2008$.

[15] E. Kerrigan and D. Mayne, "Optimal control of constrained, piecewise affine systems with bounded disturbances," IEEE Conference on Decision and Control, pp. 1552 - 1557, 2002.

[16] S. Rakovic, E. Kerrigan, and D. Mayne, "Optimal control of constrained piecewise affine systems with state-and input-dependent disturbances," International Symposium on Mathematical Theory of Networks and Systems, pp. 1 - 10, 2004.

[17] W. Zhang, J. Hu, and J. Lian, "Quadratic optimal control of switched linear stochastic systems," Systems and Control Letters, vol. 59, no. 11, pp. $736-744,2011$.

[18] L. Asselborn and O. Stursberg, "Robust control of uncertain switched linear systems based on stochastic reachability," IFAC Conference on Analysis and Design of Hybrid Systems, pp. 1 - 7, 2015.

[19] G. Calafiore and M. Campi, "The scenario approach to robust control design," IEEE Transactions on Automatic Control, vol. 51, no. 5, pp. 742-753, 2006

[20] M. Campi and S. Garatti, "The exact feasibility of randomized solutions of uncertain convex programs," SIAM Journal on Optimization, vol. 19, no. 3, pp. 1211-1230, 2008.

[21] M. Campi, S. Garatti, and M. Prandini, "The scenario approach for systems and control design," Annual Reviews in Control, vol. 33, no. 2, pp. $149-157,2009$

[22] K. Margellos, M. Prandini, and J. Lygeros, "On the connection between compression learning and scenario based single-stage and cascading optimization problems," IEEE Transactions on Automatic Control, vol. 60, no. 10, pp. 2716 - 2721, 2015.

[23] S. Formentin, F. Dabbene, R. Tempo, L. Zaccarian, and S. Savaresi, "Scenario optimization with certificates and applications to antiwindup design," IEEE Conference on Decision and Control, pp. 2810 $-2815,2014$

[24] S. Grammatico, X. Zhang, K. Margellos, P. Goulart, and J. Lygeros, "A scenario approach for non-convex control design," IEEE Transactions on Automatic Control, vol. 61, no. 2, pp. 334 - 345, 2016.

[25] P. Mohajerin, T. Sutter, and J. Lygeros, "Performance bounds for the scenario approach and an extension to a class of non-convex programs," IEEE Transactions on Automatic Control, vol. 60, no. 1, pp. $46-58,2015$.

[26] S. Boyd and L. Vandenberghe, Convex Optimization. Cambridge University Press, 2004.

[27] T. Alamo, R. Tempo, and E. Camacho, "Randomized strategies for probabilistic solutions of uncertain feasibility and optimization problems," IEEE Transactions on Automatic Control, vol. 54, no. 11, pp. $2545-2559,2009$

[28] T. Alamo, R. Tempo, and A. Luque, "On the Sample Complexity of Randomized Approaches to the Analysis and Design under Uncertainty," American Control Conference, pp. 4671 - 4676, 2010.

[29] G. Schildbach, L. Fagiano, and M. Morari, "Randomized Solutions to Convex Programs with Multiple Chance Constraints," SIAM Journal on Optimization, vol. 23, no. 4, pp. 2479 - 2501, 2013.

[30] D. Ioli, A. Falsone, and M. Prandini, "Optimal energy management of a building cooling system with thermal storage: A convex formulation," IFAC International Symposium on Advanced Control of Chemical Processes, pp. 1151 - 1156, 2015. 